\title{
Prevención del síndrome doloroso regional complejo asociado a cirugía. Una revisión de la literatura
}

\author{
Prevention of complex regional painful syndrome associated \\ with surgery. A review of the literature \\ Daniel López García (1), Antonio Córdoba-Fernández (2), \\ José María Juárez-Jiménez (3), Rafael Rayo-Rosado (4) \\ (1) DP. sistente Honorario. Departamento de Podología. Universidad de Sevilla \\ (2) DP, PhD. Profesor Titular de Universidad. Departamento de Podología. Universidad de Sevilla. \\ (3) DP, PhD. Profesor Colaborador. Departamento de Podología Universidad de Sevilla. \\ (4) DP, PhD. Profesor Colaborador. Departamento de Podología Universidad de Sevilla.
}

\author{
Correspondencia: \\ Prof. Dr. Antonio Córdoba Fernández \\ Departamento de Podología. Universidad de Sevilla. \\ Facultad de Enfermería, Fisioterapia y Podología. C/ Avicena s/n, 41009 Sevilla (España) \\ E-mail: acordoba@us.es
}

Fecha de recepción: 7 de febrero de 2011

Fecha de aceptación: 10 de abril de 2011

Los autores declaran no tener ningún tipo de interés económico o comercial.

\section{RESUMEN}

El síndrome doloroso regional complejo (SDRC) es un cuadro clínico de fisiopatología incierta y abordaje terapeútico complejo. Su etiología es diversa, se asocia con frecuencia a inmovilizaciones prolongadas tras cirugía o a traumatismos y se caracteriza por presentar una clínica variable con presencia de dolor intenso, hiperalgesia, alodinia, edema y trastornos vasomotores tróficos y óseos. Dado que no existen pruebas complementarias específicas para su diagnóstico la detección precoz y sobre todo la prevención son fundamentales para evitar secuelas. Estudios recientes han demostrado la eficacia de la vitamina $\mathrm{C}$ en la prevención del SDRC. Dada la inocuidad de su empleo y las evidencias disponibles respecto a las ventajas de su uso, la indicación de vitamina $\mathrm{C}$ en cirugía podológica de forma rutinaria debería ser considerada.

Palabras clave: Síndrome doloroso regional complejo, prevención, acido ascórbico, cirugía

\section{ABSTRACT}

Complex regional pain syndrome (CRPS) is a clinical entity of uncertain pathophysiology and complex therapeutic approach. Its varied etiology is often associated with prolonged immobilization after surgery or trauma and is characterized by a variable clinical presence of pain, hyperalgesia, allodynia and vasomotor and bone trophic changes. There are no specific laboratory tests for early diagnosis and prevention are essential to prevent damage. Recent studies have demonstrated the efficacy of vitamin $\mathrm{C}$ in the prevention of CRPS. Given the safe use and the available evidence regarding the benefits of employment, an indication of vitamin $\mathrm{C}$ in podiatric surgery should be considered routinely.

Key words: Complex regional pain syndrome, prevention, ascorbic acid, surgery

Sumario: 1. Introducción. 2. Vitamina C en la prevención del SDRC. 3. Otras sustancias para la prevención del SDRC. 4. Riesgo de SDRC asociado a cirugía. Consideraciones. 5. Conclusión. Bibliografía

Referencia normalizada: López García D, Córdoba-Fernández A, Juárez-Jiménez JM, Rayo-Rosado R. Prevención del síndrome doloroso regional complejo asociado a cirugía. Una revisión de la literatura. Rev. Int. Cienc. Podol. 2011; 5(2): 17-24. 


\section{INTRODUCCIÓN}

El motivo de esta revisión es dar a conocer al lector las características del denominado SDRC y la manera de prevenirlo en el ámbito de la cirugía podológica. Estudios recientes han demostrado la eficacia de la administración de vitamina $\mathrm{C}$ como medida profiláctica para la prevención del SDRC como complicación postquirúrgica. La vitamina $\mathrm{C}$ es un antioxidante natural que bloquea los radicales libres con lo que protege al endotelio vascular de la microangiopatía característica del síndrome. Por otro lado la vitamina $C$ juega un papel esencial en la síntesis de colágeno ya que favorece el tiempo de cicatrización cutánea y acelera la consolidación ósea tras osteotomía o fractura.

La IASP (International Association for the Study of Pain) define el Síndrome Doloroso Regional Complejo (SDRC), como variedad de condiciones dolorosas de localización regional, posteriores a una lesión, que presentan predominio distal de síntomas anormales, excediendo en magnitud y duración al curso clínico esperado del incidente inicial, ocasionando con frecuencia un deterioro motor importante, con una progresión variable en el tiempo ${ }^{1}$.

Desde su primera descripción, la enfermedad ha recibido diferentes denominaciones: causalgia, atrofia ósea inflamatoria, síndrome doloroso postraumático, distrofia simpático refleja, algodistrofia, causalgia, atrofia de Suddeck, síndrome hombro-mano, osteoporosis dolorosa idiopática del pie, algodistrofia descalcificante, etc. El subcomité de taxonomía de la IASP en 1994 pasa a denominarlo SDRC denominación con la aparece actualmente en la clasificación internacional de enfermedades (CIE). El SDRC incluye un vasto cuadro nosológico que integra a entidades patológicas variadas descritas en periodos muy diferentes. Existen dos variantes de SDRC según la etiología. El tipo I es secundario a una lesión de partes blandas o una inmovilización, mientras que el Tipo II, aparece tras la lesión de un nervio. Ambos tipos tienen los mismos signos y síntomas sin embargo, los factores desencadenantes del síndrome no siempre se presentan. En el SDRC tipo I los factores desencadenantes pueden ser variados (fracturas, esfuerzos, cirugía, contusiones, aplastamientos, esguin- ces, inmovilizaciones prolongadas, reeducación inadecuada, accidente vascular cerebral o infarto de miocardio), en ocasiones son yatrogénicos como los originados por tratamiento con fenobarbital, isoniacida, etionamida o yodo radiactivo. Los factores predisponentes pueden ser igualmente variados, metabólicos (diabetes), tabaquismo, predisposición genética (HLA-DR15, HLA-DQ1) y factores psicológicos (ansioso-depresivos). En el SDRC tipo II se presenta como antecedente obligado la lesión parcial o total de un tronco nervioso periférico o una de sus ramas terminales.

Este síndrome presenta una serie de signos y síntomas principales que incluyen alteraciones nociceptivas (dolor intenso, hiperestesia, hiperalgesia, alodinia, trastorno hemisensorial, hipoestesia), tumefacción, cambios de color, anomalías de sudoración, cambios en la piel (palidez, fibrosis, hiperqueratosis y piel brillante y fina) y alteraciones tróficas y vasomotoras. A nivel musculo-esquelético se caracteriza por atrofia muscular y ósea, edema (congestivo, blando y de aparición precoz que en las fases tardías puede hacerse más duro), disfunción motriz (temblor, distonía, mioclono, limitación de la movilidad articular y disminución de la fuerza muscular. En el SDRC II no hay una distribución anatómica, por lo tanto no es radicular ni sigue la distribución de un nervio periférico.

La fisiopatología del SDRC aún no ha sido bien definida. En los mecanismos fisiopatológicos parecen participar de un lado un factor desencadenante, de otro la neurotransmisión del impulso nervioso a centros simpáticos regionales, la percepción posiblemente distorsionada del mensaje y una respuesta neurovegetativa desmesurada en intensidad y extensión, anormalmente prolongada que desencadena una alteración regional y mantenida de la microcirculación. La hiperexcitabilidad del sistema simpático conlleva una vasoconstricción arteriolar seguida, de forma indirecta, de una dificultad metabólica en la microcirculación, que daría lugar a una vasodilatación capilar con extravasación, edema y sufrimiento celular. Este círculo vicioso es mantenido por la liberación de sustancias tóxicas. La incapacidad del sistema venoso y linfático hace que aparezca el edema con modificaciones de la sustancia fun- 
damental, que se polimeriza, y una actividad intensa de los fibrocitos. De este modo, la fase edematosa e inflamatoria da lugar a una fibrosis de importancia variable, a veces cicatricial y retráctil. La liberación de sustancias (citoquinas y cininas) desencadena la sensibilización periférica. Así mismo, hay una sensibilización central que produce alteraciones importantes a nivel autonómico, del dolor y del sistema motor.

No existe ningún signo o síntoma patognomónicos del SDRC. El diagnóstico se realiza mediante la historia clínica y la exploración. No se recomienda realizar pruebas de laboratorio ya que son inespecíficas. Es necesario pensar en la existencia del SDRC, para poder llegar a su diagnóstico y tratarlo de forma precoz y correcta, con el fin de evitar secuelas irreversibles. La radiología convencional puede mostrar, de forma tardía, desmineralización ósea subcondral de tipo geódico, osteoporosis, afectación de la sinovial, envolturas articulares y tegumentos, pseudoinflamación de partes blandas (Fig. 1 y 2).

Otras pruebas útiles en el diagnostico son la densitometría, medición del contenido mi- neral óseo por absorción fotónica, TAC, RMN, gammagrafía, exploraciones transóseas, termografía, flujometría laser-doppler y bloqueo neuronal diferencial.

Numerosos ensayos clínicos controlados y aleatorizados han mostrado los beneficios de la elección de un tratamiento $u$ otro con un alto grado de evidencia. Se puede concluir que sólo los bifosfonatos (alendronato, clodronato o palmidronato), parecen ofrecer beneficios a los pacientes con SDRC. Han sido reportadas mejorías con dimetilsulfóxido, esteroides (prednisolona o metil-prednisolona), bloqueo epidural con clonidina, baclofeno intratecal y estimulación del cordón espinal. No obstante, se puede concluir que son necesarios ensayos mejor diseñados a partir de los cuales poder extraer datos concluyentes ${ }^{2}$.

Algunos estudios parecen haber demostrado la eficacia de la gabapentina en el tratamiento del dolor asociado a SDRC. Mellick et al reportan 6 casos de SDRC tratados con gabapentina con resolución del síndrome en todos los $\operatorname{casos}^{3}$. La gabapentina es un análogo del neurotransmisor GABA (ácido gamma amino butiral) y se ha empleado como antiepiléptico,

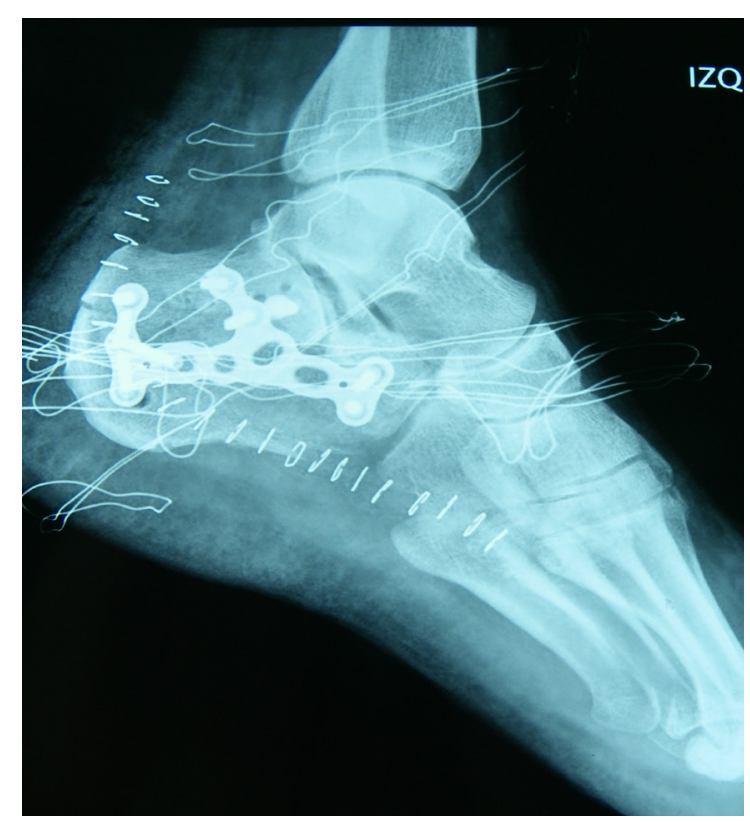

Figura 1

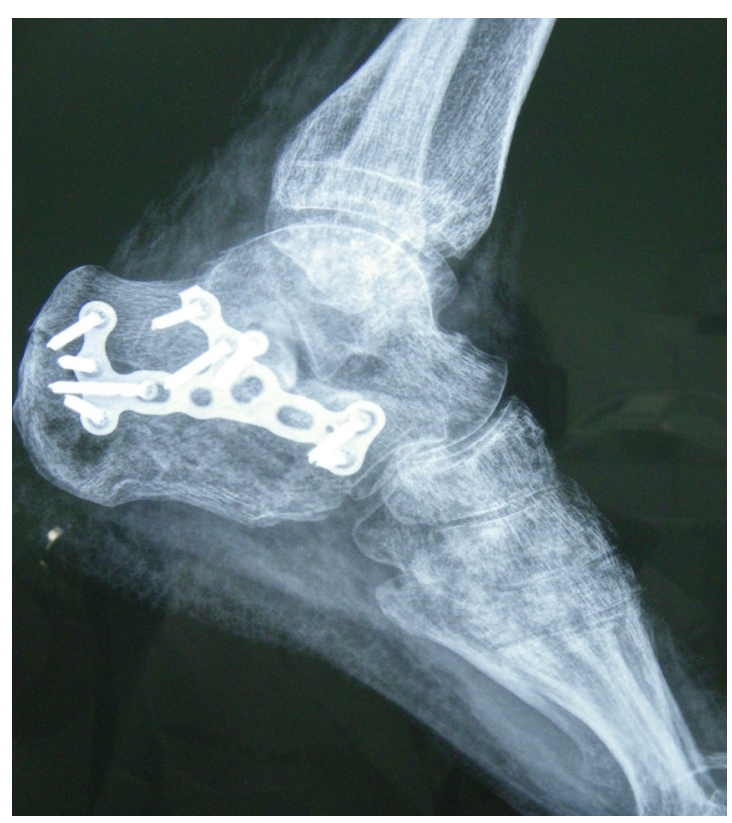

Figura 2

Figura 1 y 2. Paciente intervenido de fractura de calcáneo que fue intervenido quirúrgicamente. La imagen de la derecha corresponde al mismo paciente a los 4 meses donde se aprecian signos radiológicos típicos de SDRC. 
en el tratamiento del dolor neuropático como el ocurre en SDRC tipo I y II o en la neuropatía diabética en calcetín. Su empleo en el tratamiento del dolor asociado a SDRC parece estar justificado porque este actúa atravesando la barrera hematoencefálica e inhibiendo los receptores del dolor, también actúa frenando los cambios en la piel y en los tejidos blandos.

\section{VITAMINA C EN LA PREVENCIÓN DEL SDRC}

En la última década se han llevado a cabo estudios para analizar al efecto de la vitamina $\mathrm{C}$ en la prevención del SDRC en el marco de las fracturas. La vitamina $\mathrm{C}$ también conocida como acido ascórbico es un compuesto orgánico soluble en agua, que aunque los humanos no son capaces de sintetizarla, es un micronutriente esencial en muchos procesos enzimáticos y químicos. Actúa como un cofactor de ocho enzimas diferentes, implicadas en la hidroxilación del colágeno, biosíntesis de la carnitina y norepinefrina, hormonas peptídicas y metabolismo de la tirosina. Además es un potente antioxidante.

En la literatura existen varios ensayos clínicos controlados aleatorizados que han demostrado que la vitamina $\mathrm{C}$ reduce la incidencia del SDRC después de fracturas. Besse et al. ${ }^{4}$ llevaron a cabo un estudio cuasi-experimental comparando 2 grupos sometidos a cirugía del pie y tobillo, uno sin tratamiento y otro con administración de 1 gramo de vitamina $\mathrm{C}$ diaria. De un total de 420 pies de 392 pacientes (185 en el grupo control y 235 en el grupo estudio), el SDRC ocurrió en 18 casos del grupo control $(9,6 \%)$ y 4 casos en el grupo estudio $(1,7 \%)$. El factor psicológico (ansioso-depresivo), mostró un incremento de la prevalencia del SDRC, concretamente el $36,3 \%$ de los afectados por el SDRC. La hipótesis de su eficacia es que la vitamina $\mathrm{C}$ bloquea los radicales libres y de ese modo protege el endotelio capilar.

Por otro lado algunos estudios han demostrado que los radicales libres obstaculizan la microcirculación. Van der Laan et al..$^{5}$ encontraron que los radicales libres originaban la alteración de la fibra muscular en el SDRC I, causando la oxidación de los lípidos de la membrana y dando lugar a alteraciones capilares que se traducían en microangiopatía; contribuyendo a la fisiopatología del síndrome. Los autores postulan que la vitamina $C$ es un antioxidante natural que actúa bloqueando los radicales libres con la consiguiente protección del endotelio capilar. Zollinger et al. ${ }^{6}$ Estos mismo autor con otros colaboradores en un ensayo clínico aleatorizado multicéntrico a doble ciego controlado con placebo, llevado a cabo sobre 427 fracturas de muñeca (416 pacientes) administró placebo o vitamina $\mathrm{C}$ a dosis de 200,500 o $1500 \mathrm{mg}$. a cada uno de los grupos durante 50 días. En el estudio se analizó el efecto según sexo, edad, tipo de fractura y quejas relacionadas con la inmovilización con escayola en la aparición del SDRC. La prevalencia de SDRC fue de un $2,4 \%$ en el grupo de la vitamina C y de $10,1 \%$ en el grupo placebo con diferencias significativas $(\mathrm{P}=0.002)$. Todos los pacientes afectados fueron mujeres mayores, lo que sugiere que el estatus de estrógenos podría ser un factor predisponente $\mathrm{El}$ análisis de las diferentes dosis de vitamina $\mathrm{C}$ mostró que la prevalencia de SDRC fue de un $4,2 \%$ en el grupo que recibió $200 \mathrm{mg}$, $1,8 \%$ en el grupo que recibió $500 \mathrm{mg}$ y $1,7 \%$ en el que recibió $1500 \mathrm{mg}$. Los autores concluyen que la administración de vitamina $\mathrm{C}$ reduce la prevalencia de SDRC tras fracturas de muñeca y recomiendan una dosis de 500mg durante 50 días.

La farmacodinamia de la vitamina $\mathrm{C}$ en el tratamiento de las fracturas o prevención del SDRC no es del todo conocida. Estudios previos han mostrado un efecto positivo en el proceso de cicatrización. Yilmaz et al. ${ }^{7}$, en un estudio llevado a cabo con ratas, sugirió que la vitamina $\mathrm{C}$ aumentaba la cicatrizacion de la fracturas y Sarisozen et al. ${ }^{8}$ observaron que la vitamina $\mathrm{C}$ disminuye el tiempo inflamatorio favorecedor del SDRC.

Por otro lado, la isquemia producida por el torniquete durante la cirugía, causa daños en el endotelio y subendotelio debido a los oxidantes neurotrófilos. Kearns et al. ${ }^{9}$ observaron que la administración de vitamina $\mathrm{C}$ protegía de este daño a las ratas posiblemente por la disminución de la actividad neurotrofila respiratoria. Los autores también evidenciaron el papel antioxidante de la vitamina $C$ en el daño al músculo esquelético causado por el síndrome 
compartimental post-torniquete. Los autores postulan que la vitamina $\mathrm{C}$ puede prevenir la disfunción microvascular y la microangiopatía de una reacción inflamatoria como la ocurrida en el SDRC. En otro estudio, Zollinger et al. ${ }^{10}$ realizaron un ensayo a doble ciego placebo controlado en 115 adultos con al menos una fractura de muñeca. Cincuenta y dos pacientes (54 fracturas) recibieron vitamina $\mathrm{C}$ y 63 pacientes (65 fracturas) recibieron placebo. Tras un seguimiento de un año, el SDRC ocurrió en 4 (7\%) muñecas en el grupo de la vitamina C y 14 (22\%) en el grupo placebo encontrándose diferencias significativas. Este mismo autor con otros colaboradores realiza un estudio prospectivo de cohortes en 27 pacientes afectados de artritis en la $1^{\mathrm{a}}$ articulación trapecio-metacarpiana de la mano grado II y III sometidos a una artroplastia y colocación de prótesis. Este tipo de cirugía ha mostrado tener muchas probabilidades de desencadenar un SDRC tipo 1. El tratamiento preventivo y postquirurgico consistió en tratamiento con AINES y vitamina $\mathrm{C}$ (se administraron $500 \mathrm{mg}$ de vitamina $\mathrm{C}$ diaria, 2 días antes de la intervención y 50 días posteriores a la misma) asociado a ejercicios de circunducción y oposición sin movilización pasiva. Se realizó seguimiento a los 5 días y a las 2 semanas con radiografías de control a los 3, 6 y 12 meses. Se estudió la coloración, temperatura, e inflamación de la extremidad. Los pacientes fueron analizados mediante una escala visual análoga en satisfacción, dolor y actividad pre y postoperatoria. Los resultados fueron que no se produjo ningún caso de SDRC $^{11}$.

Apurva et al. ${ }^{12}$ realizaron un ensayo con 416 pacientes con 427 fracturas de muñeca, estos fueron aleatorizados para recibir un tratamiento con placebo o con 200, 500 o $1500 \mathrm{mg}$ de vitamina $\mathrm{C}$ al día, por un periodo de 50 días. La prevalencia del SDRC tipo I fue de $10 \%$ en el grupo placebo y de $2,4 \%$ en el grupo de la vitamina $\mathrm{C}$. La dosis que produjeron mejores resultados para la prevención del SDRC fue la de $500 \mathrm{mg}$. No se encontraron diferencias entre el grupo placebo y el grupo que recibió $200 \mathrm{mg}$, lo que sugiere que una dieta normal (5 piezas de fruta y verdura que aportan 210-280 $\mathrm{mg}$ de vitamina C) no es suficiente para la prevención del SDRC. Por lo general los efectos adversos derivados de un aporte excesivo de vitamina $\mathrm{C}$ son diarrea e hinchazón abdominal. Estos pueden ser evitados con dosis inferiores a $1000 \mathrm{mg}$ diarios en personas sanas. Los autores sugieren que la vitamina $\mathrm{C}$ evita la producción de radicales libres que intervienen en la aparición del SDRC. También destacan su papel como antioxidante en el daño del músculo esquelético derivado del uso del torniquete y del síndrome compartimental, en la formación de colágeno y cicatrización de hueso. Actúa además bloqueando los radicales libres procedentes de la inflamación tras la fractura con la consiguiente protección del endotelio capilar. Kermad et al. ${ }^{13}$ realizaron un estudio prospectivo con 185 pacientes afectados de fracturas de radios distales de muñeca tratados con reducción cerrada y aguja. El primer grupo de 100 pacientes se sometió al procedimiento sin prescripción de vitamina $\mathrm{C}$ y el segundo grupo de 85 pacientes recibió 1 gr de vitamina $C$ cada mañana durante 45 días. En el primer grupo hubo 9 pacientes $(9 \%)$ que desarrollaron SDRC mientras que en el segundo grupo sólo 2 pacientes $(2,35 \%)$ lo desarrollaron.

\section{OTRAS SUSTANCIAS PARA LA PRE- VENCIÓN DEL SDRC}

Algunas investigaciones han analizado la eficacia de algunas sustancias como la calcitonina en la prevención del SDRC. Esta sustancia se ha utilizado con éxito en el tratamiento del SDRC. Riou et al. ${ }^{14}$ realizaron un estudio a doble ciego aleatorizado en 91 pacientes en los que se comparó la eficacia de la tirocalcitonina en la prevención del SDRC. Se administró un placebo a 40 pacientes y tirocalcitonina a 51 pacientes en diferentes intervenciones quirúrgicas: 10 fracturas de radios en la muñeca que requirieron fijación externa, 34 intervenciones ortopédicas varias, 25 intervenciones de rodilla (ligamentoplastia) y 22 intervenciones de pies (HAV, artrodesis, y fracturas). En el grupo placebo se produjeron 5 casos de SDRC $(12,5 \%)$, y en el grupo de tirocalcitonina obtuvieron 4 casos $(7,8 \%)$. El estudio no mostró diferencias significativas entre los grupos. Los autores concluyen que si bien el medicamento se muestra eficaz como tratamiento del SDRC 
este no resulta eficaz como tratamiento preventivo. Algunos ensayos clínicos como el llevado a cabo por Kissling et al. ${ }^{15}$ han demostrado que el uso de la calcitonina resulta eficaz en el prevención de la recidiva del SDRC. El estudio se llevó a cabo en 2 grupos de pacientes todos ellos con síntomas de SDRC o con historia de esta enfermedad. A 18 pacientes se les administró calcitonina (100 IU subcutáneo o en spray nasal) 4 días antes y 23 días tras cirugía o tratamientos conservadores y 74 pacientes con SDRC tras cirugía o tratamientos conservadores a los que no se les administró ningún tratamiento. En el grupo de la calcitonina hubo solo 1 paciente (3\%) que desarrolló SDRC y en el grupo control 21 pacientes (28\%) desarrollaron un SDRC. La calcitonina posee un efecto anti-osteoclastico, lo que favorece la acción de los osteoblastos y acelera el tiempo de cicatrización ósea. También posee un efecto analgésico a nivel central disminuyendo la actividad de ciertas células en la hipófisis e hipotálamo. A nivel periférico inhibe la síntesis de prostaglandinas y posee un efecto vasodilatador (se ha usado para tratar el síndrome de Raynaud). La calcitonina parece interrumpir los factores favorecedores del SDRC (dolor, tiempo de fractura y conflicto arteriovenoso). El estudio concluye que en pacientes con historia de SDRC está recomendada la administración de calcitonina para evitar la recidiva de la enfermedad en casos de reintervención de cirugía ortopédica o en casos en que se sometan a tratamientos conservadores después de un traumatismo. Marx et $a l .{ }^{16}$ realizaron un estudio de serie de casos para examinar la recurrencia en pacientes con historia de SDRC después de la cirugía y reintervenidos en la misma localización a los que se les aplicó un protocolo de intervención perioperatorio que incluía una serie de medidas preventivas (reducir al máximo los tiempos quirúrgicos con aplicación de torniquete, administración de tratamiento del dolor postoperatorio y movilización precoz asociado a la administración de 100 UI de calcitonina diariamente dos días antes de la cirugía y dos semanas después. De los 10 pacientes que participaron en el estudio ninguno desarrolló SDRC cuando lo esperado era una recidiva de un 50\% de los pacientes reintervenidos. Riou lo usó en pacientes sin historia de SDRC y Kissling en pacientes reinterve- nidos pero en otras localizaciónes. Los autores concluyen que el protocolo con calcitonina resulta útil en pacientes en los que el empleo de la misma para el tratamiento del síndrome en el pasado resultó eficaz y destacan que el resto de medidas profilácticas favorecen el mantenimiento del rango de movimiento articular y controlar el edema periférico por lo que resulta útil para frenar el círculo vicioso que se produce el SDRC.

Matayoshi et $a l^{17}$ realizaron un estudio controlado en pacientes con hemiplejía grave después de un accidente cerebrovascular para demostrar el uso de la calcitonina en la prevención del SDRC. El grupo control (35 pacientes) fue tratado con rehabilitación 2 horas seis veces por semana, incluyendo terapia física, terapia ocupacional, fisioterapia, y movimiento de la extremidad afecta. El grupo de la calcitonina (24 pacientes) se le administró 20 unidades de elcatonin ${ }^{\circledR}$ (calcitonina), una vez en semana, además de rehabilitación. La incidencia del SDRC fue mas baja en el grupo de la calcitonina, 3 de 24 pacientes $(12,5 \%)$ que en el grupo control 20 de 35 pacientes (57,1\%). El inicio del SDRC en el grupo de la calcitonina fue completamente evitado cuando se comenzó el tratamiento 4 semanas después del accidente cerebrovascular, pero los efectos preventivos eran débiles cuando se empezó 6 semanas después. Los autores justifican sus resultados basándolos en que en la fase temprana del SDRC se producen fuertes desajustes en los reflejos nerviosos autónomos que ocurren como compensación del drástico enlentecimiento del flujo sanguíneo muscular, y esto puede estar asociado con el inicio del síndrome. En los pacientes con hemiplejía la actividad nerviosa simpática del músculo se ve aumentada, mientras la actividad nerviosa autonómica en la piel es atenuada. La mayoría de los casos de SDRC se originan como consecuencia de una disfunción del sistema nervioso central como el que ocurre en los pacientes con hemiplejia. El estudio concluye que la calcitonina es útil en dolor neuropático, mejora la autonomía de los reflejos y el flujo sanguíneo en pacientes con SDRC y parece mejorar la función anormal de las fibras $\mathrm{C}$ (sensibilidad periférica), aunque no se han hecho estudios concluyentes al respecto. Los autores sugieren que la calcitonina intramuscu- 
lar suprime el inicio del SDRC en pacientes con hemiplejía.

\section{RIESGO DE SDRC ASOCIADO A CIRUGÍA. CONSIDERACIONES}

Schuind et al..$^{18}$ en un artículo de revisión acerca de la prevención del SDRC tras cirugía de la mano plantean una pauta de prevención consistente en:

1. Considerar la predisposición del paciente y evitar la cirugía si es posible en pacientes con predisposición al SDRC, ya que hay una posibilidad de un $15 \%$ de recidiva.

2. La cirugía debe realizarse evitando la inflamación postoperatoria. El proceso debería ser lo mas atraumático posible. Evitar el hematoma postoperatorio.

3. Realizar desbridamiento y saneamiento de la herida. Si hay infección o si se va a usar osteosíntesis de fijación se aconseja usar antibióticos.

4. Es esencial favorecer el retorno venoso en el postoperatorio inmediato, para disminuir la presión venosa y con ello el edema.

5. Promover la deambulación temprana del paciente, para ello la osteosíntesis debe ser estable.

6. Evitar efecto torniquete con el vendaje en el postoperatorio, ya que con ellos impediríamos el retorno venoso.

7. Son importantes los analgésicos postoperatorios, ya que al no doler disminuye el efecto psicológico no deseado de la cirugía. En el caso de fracturas de radios distales una infiltración intraarticular con morfina disminuye el riesgo de SDRC.

8. Reconocer los dolores, que algunas veces aparecen, causados por distensión de los tejidos blandos (edemas)

9. Tratamiento psicológico de la ansiedad e inquietud.

Todas estas medidas se explican en base a reducir los factores que incrementan el riesgo de desarrollar un SDRC (circulo vicioso formado por vasodilatación arterial, conflicto arteriovenoso, bajo flujo capilar, incremento de la presión venosa, inapropiada respuesta vasomotora y persistencia de la estimulación de los nociceptores). La inmovilización y el incremento de la presión venosa mantienen el estado de bajo flujo capilar visto en el SDRC. La inmovilización puede deberse al intenso dolor o a la osteosíntesis y el incremento de la presión venosa puede deberse a vendajes constrictivos o a que el paciente no eleva la extremidad, lo que contribuye al círculo vicioso. Por ello resulta esencial la movilización temprana así como las medidas que favorezcan el retorno venoso. No obstante existen individuos predispuestos a desarrollar un SDRC, se ha observado que aquellos pacientes que usan su mano aun intervenida quirúrgicamente y mantienen un buen flujo sanguíneo tienen menos riesgo de desarrollar un SDRC que los que no la usan porque son más sensibles al dolor. Existen también individuos más sensibles que liberan más mediadores vasoactivos y radicales libres y por tanto están más predispuestos. En cualquier caso cuando el síndrome aparece, los cambios en la microcirculacion deberían ser reversibles mediante movilización temprana. Por otro lado la analgesia postoperatoria permite la movilización precoz del miembro reduciendo el riesgo de desarrollar complicaciones.

\section{CONCLUSIÓN}

Los resultados de mayoría de estudios existentes apoyan el uso de la vitamina $\mathrm{C}$ en la profilaxis del SDRC tras cirugía del pie o de la muñeca. La administración de vitamina $\mathrm{C}$ por vía oral a dosis de $500 \mathrm{mg} 2$ días antes y 50 días después de la cirugía o tratamiento conservador en fracturas ha mostrado ser más eficaz y costo-efectiva en la prevención del SDRC que otros tratamientos (calcitonina). Aunque la fisiopatología del síndrome no es del todo bien conocida lo cierto es que el efecto beneficioso de la vitamina $\mathrm{C}$ en la prevención del SDRC parece radicar en varios aspectos. Por un lado bloquea los radicales libres y de ese modo protege el endotelio capilar, impidiendo la microangiopatía que acompaña al síndrome. Al margen de los beneficios de la vitamina $\mathrm{C}$ en la profilaxis del SDRC su efecto beneficioso se asocia además el hecho de que actúa favoreciendo la síntesis de colágeno y por tanto el proceso de cicatrización cutánea y ósea acelerando la con- 
solidación de la fracturas u osteotomías. Actúa además disminuyendo el tiempo de la fase inflamatoria aguda de la cicatrización con lo que previene la disfunción microvascular. Su papel como antioxidante previene además el daño al músculo esquelético causado por el síndrome compartimental postorniquete. Concluimos por tanto que la administración rutinaria de vitamina $\mathrm{C}$ después de procedimientos quirúrgicos en el pie resulta razonablemente útil a la vista de las evidencias disponibles. Esta medida preventiva asociada a otras medidas (control de los tiempos quirúrgicos, empleo racional de antibióticos, cirugía mínimamente traumática y movilización precoz asociada a terapia física), además de reducir el riesgo de SDRC favorece la recuperación del paciente y reduce las complicaciones asociadas a la práctica quirúrgica. Por tanto consideramos que la inocuidad de su empleo asociado a las ventajas de su costo-efectividad, justifican sobradamente la indicación rutinaria de vitamina $C$ en cirugía podológica especialmente en los procedimientos osteo-articulares.

\section{BIBLIOGRAFÍA}

1. Neira F, Ortega J L. El síndrome doloroso regional complejo y medicina basada en la evidencia. Rev Soc Esp Dolor 2007; 2: 133-146.

2. Tran de QH, Duong S, Bertini P, Finlayson RJ. Treatment of complex regional pain syndrome: a review of the evidence. Can J Anaesth 2010; 57: 149-66.

3. Mellick GA, Mellick LB. Reflex sympathetic dystrophy treated with gabapentin. Arch Phys Med Rehabil 1997; 78:98-105.

4. Besse JL, Gadeyne S, Galand-Desmé S, Lerat JL, Moyen B. Effect of vitamin C on prevention of complex regional pain syndrome type I in foot and ankle surgery. Foot Ankle Surg 2009; 15: 179-82.

5. Van der Laan L, Kapitein PJ, Oyen WJ, Verhofstad AA, Hendriks T, Goris RJ. A novel animal model to evaluate oxygen derived free radical damage in soft tissue. Free Radic Res 1997;26: 363-72.

6. Zollinger PE, Tuinebreijer WE, Breederveld RS, Kreis RW. Can Vitamin C prevent complex regional pain syndrome in patients with wrist fractures? A randomized, controlled, multicenter dose-response study $J$ Bone Joint Surg Am 2007; 89: 1424-31. http://dx.doi.org/10.2106/JBJS.F.01147

7. Yilmaz C, Erdemli E, Selek H, Kinik H, Arikan M, Erdemli B. The contribution of vitamin C to healing of experimental fractures. Arch Orthop Trauma Surg 2001;121: 426-8. http://dx.doi.org/10.1007/s004020100272

8. Sarisozen B, Durak K, Dincer G, Bilgen OF. The effects of vitamins E and C on fracture healing in rats. J Int Med Res 2002;30: 309-13.

9. Kearns SR, Daly AF, Sheehan K, Murray P, Kelly C, Bouchier-Hayes D. Oral vitamin C reduces the injury to skeletal muscle caused by compartment syndrome. J Bone Joint Surg Br 2004;86: 906-11. http://dx.doi. org/10.1302/0301-620X.86B6.14177

10. Zollinger PE, Tuinebreijer WE, Kreis RW, Breederveld RS. Effect of vitamin C on frequency of reflex sympathetic dystrophy in wrist fractures: a randomised trial. Lancet 1999; 354: 2025-8. http://dx.doi.org/10.1016/ S0140-6736(99)03059-7

11. Zollinger PE, Ellis ML, Unal H, Tuinebreijer WE. Clinical outcome of cementless semi-constrained trapeziometacarpal arthroplasty, and possible effect of Vitamin C on the occurrence of complex regional pain syndrome. Acta Orthop Belg 2008; 74: 317-22.

12. Shah AS, Verma MK, Jebson PJ. Use of oral vitamin C after fractures of the distal radius. J Hand Surg Am 2009; 34: 1736-8.

13. Cazeneuve JF, Leborgne JM, Kermad K, Hassan Y. Vitamin C and prevention of reflex sympathetic dystrophy following of the distal radius fracture. Acta Orthop Belg 2002;65:481-4. http://dx.doi.org/10.1016/j. jhsa.2009.08.013

14. Riou C, Daoui Y, Langlais F, Pawlotsky Y, Cheverry C. L'algodystrophie en milieu chirurgical peut-elle etre prévenue par la thyrocalcitonine? Rev Chir Orthop 1991; 77: 208-10.

15. Kissling R.O, Bloesch A-C, Sager M, Dambacher M.A, Schreiber A. Prévention de la récidive d'une maladie de Sudeck par la calcitonine. Rev Chir Orthop 1991; 77: 562-7.

16. Marx C, Wiedersheim P, Michel BA, Stucki G. Preventing recurrence of reflex sympathetic dystrophy in patients requiring an operative intervention at the site of dystrophy alter surgery. Clin Rheumatol 2001; 20: 114-8. http://dx.doi.org/10.1007/s100670170080

17. Matayoshi S, Shimodozono M, Hirata Y, Ueda T, Horio S, Kawahira K. Use of calcitonin to prevent complex regional pain syndrome type I in severe hemiplegic patients after stroke. Disabil Rehabil 2009; 31: 1773-9. http://dx.doi.org/10.1080/09638280902795573

18. Schuind F, Burny F. Can algodystrophy be prevented after hand surgery? Hand Clin 1997; 13: 455-76. 